\title{
Observations of Diffuse Emission from the Hot ISM
}

\author{
W. T. SANDERS ${ }^{1,2}$ AND R. J. EDGAR ${ }^{1,2}$ \\ ${ }^{1}$ Department of Physics, University of Wisconsin-Madison, \\ 1150 University Avenue, Madison, WI 53706, USA \\ ${ }^{2}$ Space Science \& Engineering Center, University of Wisconsin-Madison, \\ 1225 W. Dayton Street, Madison, WI 53706, USA
}

The Diffuse X-ray Spectrometer (DXS) is a Bragg-crystal spectrometer designed to obtain spectra of the diffuse $x$-ray background in the 83-44 $\AA(150-284 \mathrm{eV})$ range, with $\sim 3 \AA$ spectral resolution (10-25 eV), and $\sim 15^{\circ}$ angular resolution. It was flown successfully as an attached Shuttle payload on the STS 54 mission of NASA's Space Shuttle Endeavour in January 1993, and spectra were obtained from the diffuse background along an arc extending roughly along the galactic plane from longitude $150^{\circ}$ to longitude $300^{\circ}$. The primary conclusions so far from the analysis of the DXS data are: (1) The spectra of the diffuse background in the 83-44 $\AA$ range show emission lines or emission-line blends, indicating that the emission is thermal. Although most models of this emission have assumed that it is of thermal origin, this is the first detection of lines in the diffuse background in this wavelength range. (2) The detected spectra do not resemble the model spectra of cosmic abundance equilibrium plasmas at any temperature in the $10^{5}-10^{7} \mathrm{~K}$ range. This is independent of the particular plasma model used, Raymond \& Smith, Mewe \& Kaastra, or Monsignori-Fossi \& Landini. (3) The detected spectra do not resemble the model spectra of depleted abundance equilibrium plasmas at any temperature in the $10^{5}-10^{7} \mathrm{~K}$ range, for a variety of assumed elemental depletions and the same emission models. This aspect of the analysis is not completed. (4) Tentative line identifications can be made, but other lines predicted to arise from the same ions must be of consistent strength in both the DXS and EUVE data sets.

\section{Introduction}

In this talk, we discuss the soft x-ray observations of the hot interstellar medium (ISM). The first part summarizes the spatial structure of the ISM as revealed by sounding rocket and satellite experiments. The second part summarizes spectral results, in particular, the data from the Diffuse X-ray Spectrometer (DXS) experiment that our group built and that NASA flew as an attached Shuttle payload in 1993.

A basic assumption in all this is that observations of the low energy $\mathrm{x}$-ray diffuse background are in fact observations of x-rays emitted by the hot ISM. The implied picture then is that the solar system is surrounded by a $10^{6} \mathrm{~K}$ interstellar plasma. We will address the temperature more later, but it is approximately correct. Further, we know that the local ISM is relatively deficient in neutral material, which would easily absorb low energy $\mathrm{x}$-rays, out to distances of 50-100 pc in all directions. So the natural home for this surrounding $x$-ray emitting plasma in within this "local cavity."

All-sky surveys of the diffuse background have been done in the low energy $x$-ray band from $160-284 \mathrm{eV}$ (44-80 $\AA$, also known as the carbon band, the $\mathrm{C}$ band, and the $\frac{1}{4} \mathrm{keV}$ band) by a series of sounding rockets (McCammon et al. 1983), by the $S A S$ \& satellite (Marshall \& Clark 1984), by the HEAO 1 satellite (Garmire et al. 1992), and by the ROSAT satellite (Snowden et al. 1995). One all-sky survey has also been done in the $120-188 \mathrm{eV}$ band (66-100 $\AA$, also known as the boron band, or the $\mathrm{B}$ band) by a series of sounding rockets (McCammon et al. 1983), and there are $\sim 20$ pointings around the 
sky in a band from $70-111 \mathrm{eV}(112-177 \AA$, also known as the beryllium band or the Be band) from sounding rockets (Bloch et al. 1986) (Juda et al. 1991) (Edwards 1990).

When the count rates from all four $\frac{1}{4} \mathrm{keV}$ band experiments are compared, with allowances made for the different instrumental response functions, they agree with each other to better than $10 \%$ (Snowden et al. 1995). This tells us that the features seen on the maps are real, and that they have persisted over a twenty-year time interval. The first observations of the soft x-ray background (Bowyer, Field, \& Mack 1968) have features that also are apparent on today's maps, as can be seen if Bowyer's map is compared to the ROSAT $\frac{1}{4} \mathrm{keV}$ band map (Snowden 1993).

\section{Spatial Structure of the Local Hot ISM}

When one compares the $\frac{1}{4} \mathrm{keV}$ ROSAT map (Snowden 1993) to maps of 21-cm data (Dickey \& Lockman 1990), the most striking overall effect is the general "anticorrelation" of the two maps. The x-ray intensity is lowest in the Galactic plane and increases by a factor of four or five in some high latitude directions.

For $x$-rays in the $\frac{1}{4} \mathrm{keV}$ band, one absorption optical depth is $\sim 1 \times 10^{20} \mathrm{~cm}^{-2}$. Thus the interstellar galactic gas is opaque at low Galactic latitudes, $|b| \lesssim 30^{\circ}$, while its transmission rises to $\sim 50 \%$ at high Galactic latitudes in the directions of lowest $N_{\mathrm{H}}$. The finite intensity of the $\mathrm{x}$-ray background seen at low latitudes must be Galactic in origin and originate relatively near to the Sun, within the closest $\sim 1-2 \times 10^{20} \mathrm{~cm}^{-2}$. This corresponds to a distance of roughly $100 \mathrm{pc}$, plus or minus a factor of 2 . At the high Galactic latitudes there is a significant fraction of the sky, 15\%, where the total $N_{\mathrm{H}} \lesssim 2 \times 10^{20} \mathrm{~cm}^{-2}$, and contributions from extragalactic or halo sources are possible in addition to the local emission.

Prior to the launch of ROSAT, the data did not require that the hot interstellar medium have more than one component, the emission from the local hot plasma located in the local cavity. This component is required by the finite intensity in the Galactic plane. It had been found that simple halo models, models in which a Galactic halo exhibited symmetries about the Galactic center and Galactic plane and whose soft x-ray emission was smoothly distributed, did not work very well, so many thought that the halo emission was negligible. The higher measured count rate of the diffuse background at high latitudes was taken to be due to a greater physical extent of the emitting region in those directions, plus some small contribution from extragalactic sources (Snowden et al. 1990). One advantage of this picture was that of simplicity: no more components were introduced than were absolutely necessary. Another advantage was that it naturally explained why the $\mathrm{B}$ band map looked so similar to the $\mathrm{C}$ band maps, and why the $\mathrm{Be}$ band count rates tracked the $B$ band count rates so well even though the column density for unit optical depth in the $\mathrm{Be}$ band was less than $10 \%$ of that in the $\mathrm{C}$ band. The implication was that observed intensity differences were not due to absorption.

$R O S A T$ demonstrated that this view was wrong. The arcminute angular resolution of $R O S A T$ and its high sensitivity to diffuse emission features made possible for the first time the detection in the $x$-ray diffuse background of "shadows"-the absolption of distant soft $x$-ray emission by foreground clouds of neutral material. Soft x-ray emission from the Galactic halo was demonstrated by the detection of absorption of the soft x-ray background in Draco (Snowden et al. 1991) (Burrows \& Mendenhall 1991) by a cloud at roughly $\left(l \sim 90^{\circ}, b \sim 40^{\circ}\right)$ whose distance was found to be more than $300 \mathrm{pc}$, well outside the accepted boundary of the local cavity. Half of the x-ray background count rate in this direction is from the halo or beyond, and half is local emission, originating closer than the Draco cloud. This local emission is still twice the value typically found in 
the galactic plane, so the local emission region may extend twice as far in this direction as it does in the plane. Shadows were also seen in Ursa Major (Snowden et al. 1994), both toward an intermediate negative velocity cloud complex at a distance of $300-400 \mathrm{pc}$ (Benjamin et al. 1995), as well as towards low velocity clouds whose distances are not yet known. About $70 \%$ of the count rate in this direction originates closer than the clouds, with an intensity almost three times that typically found in the Galactic plane, while $30 \%$ comes from the halo or beyond. In this low $N_{\mathrm{H}}$ direction, the extragalactic component accounts for $20 \%$ of the total rate, leaving $~ 10 \%$ as halo emission (Snowden et al. 1994). The intensity of the halo emission in this directions appears to change by a factor of two on angular scales as small as $2^{\circ}$.

In summary, the count rate due to the halo emission can be as large as $10-50 \%$ of the total count rate in some high latitude directions where (a) the $N_{\mathrm{H}}$ is very low (Ursa Major), or (b) the halo is very bright (Draco), but the local component is still required and its intensity at high latitudes is $2-3$ times greater than its intensity in the plane.

Evidence that the local component is truly local comes from the ROSAT observation of MBM 12 (Snowden, McCammon, \& Verter 1993), a nearby (d $\$ 65$ pc) molecular cloud at intermediate latitude $\left(l \sim 159^{\circ}, b \sim-34^{\circ}\right)$. Toward this cloud, practically no shadow was seen, implying that this cloud is at the edge of the local emission region and that almost all the emission in this direction originates closer than $65 \mathrm{pc}$. These authors use this observation to determine a pressure for the hot interstellar medium of $1.25 \times 10^{4}$ $\mathrm{K} \mathrm{cm}^{-3}$. They assume that the plasma generating the local emission is described by a Raymond \& Smith equilibrium model at a temperature near $10^{6} \mathrm{~K}$. Data from the DXS and $E U V E$ spectrometers do not support this assumption, so while the pressure is likely near $10^{4} \mathrm{~K} \mathrm{~cm}^{-3}$, the exact value is not determined by this method.

Figure 1 is a schematic diagram of some of the major features of the local interstellar medium relevant to the emission of the soft $\mathrm{X}$-ray background. The view is a cut taken along longitudes $160^{\circ}-340^{\circ}$ and passing through the north and south Galactic poles. It illustrates some of the count rates and spatial geometries discussed above. Count rates are indicated in units of $10^{-6}$ ROSAT counts $\mathrm{s}^{-1} \operatorname{arcmin}^{-2}$ (SXU). Count rates after absorption are indicated in parentheses.

\section{Spectroscopic Observations of the Hot Interstellar Medium}

Until very recently, spectroscopic observations of the soft $\mathrm{x}$-ray diffuse background, which was presumed to originate in the ISM, had the limited spectral resolution of proportional counters for the energy range below $284 \mathrm{eV}$. From proportional counters, spectral information comes from the shape of the distribution of pulse heights within a given band, and from the ratio of count rates from one band to another. Over the past 20 years, it has been frequently noted that the observed pulse height distributions and band count rate ratios of the diffuse background are consistent with those predicted from a plasma in ionization equilibrium at a temperature near one million degrees, independent of the particular model used. Since there were no viable non-thermal mechanisms, and there were reasons to think that the diffuse background should have a thermal origin (e.g., supernova remnants), the standard interpretation of the diffuse background spectra became that it originated from a million degree plasma in the ISM, the hot ISM.

In January 1993, NASA flew the Diffuse X-ray Spectrometer (DXS), built by our group at the University of Wisconsin, as an attached shuttle payload. DXS is a Bragg-crystal spectrometer designed to obtain spectra of the diffuse x-ray background in the 83-44 $\AA$ (150-284 eV) range, with $\sim 3 \AA$ spectral resolution $(10-25 \mathrm{eV})$, and $\sim 15^{\circ}$ angular resolution. The primary objective of this experiment was to find out if there were lines 


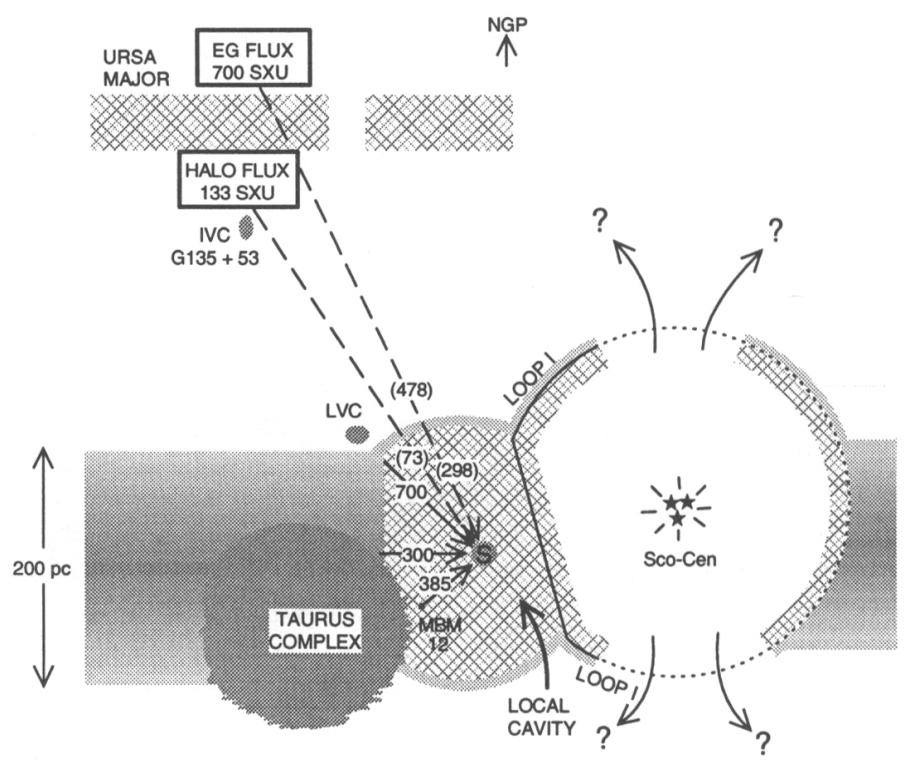

FIGURE 1. Schematic diagram of some major features of the local interstellar medium relevant to the emission of the soft X-ray background. The view is a cut taken through longitudes $160^{\circ}$ $-340^{\circ}$. Numbers shown are count rates in different directions in units of SXU, defined in the text.

in the spectrum of the diffuse background. If lines were present, then the emission has a thermal origin and the DXS experiment could answer additional questions about the emission:

- Is the plasma in coronal equilibrium?

- If so, what is the temperature?

- If not, which non-equilibrium model(s) fit the data?

- What are the wavelengths and fluxes of the dominant lines?

- What are the likely $x$-ray emitting elements and their ionization states?

- Are spectral differences apparent from one part of the sky to another?

DXS uses a curved crystal panel to Bragg-reflect incident soft x-rays towards a collimated position-sensitive proportional counter. The collimator allows each position along the counter to "see" only a restricted piece of the curved crystal panel, and each position sees its piece of the crystal panel at a different angle. Thus, each position along the counter sees a different wavelength $\mathrm{x}$-ray from a different direction on the sky than does its neighboring position. By rotating the counter about an axis perpendicular to the dispersion direction of the crystals, full wavelength coverage over the 83-44 $\AA$ range is obtained from the arc of the sky along which the counter scans. The DXS detector is described more fully elsewhere (Sanders et al. 1992).

The calibration of the DXS detectors has determined that the effective area-solid angle product rises from $\sim 0.01 \mathrm{~cm}^{2}$ sr at $151 \mathrm{eV}$, to $\sim 0.02 \mathrm{~cm}^{2}$ sr at $183 \mathrm{eV}$, and falls again to $\sim 0.01 \mathrm{~cm}^{2} \mathrm{sr}$ at $277 \mathrm{eV}$. The spectral resolution (FWHM) is $\varsigma 3 \AA$ over the full spectral range of the detectors. During the data collection times of the shuttle mission, the Orbiter was oriented such that the DXS detectors repeatedly scanned the same arc 
on the sky-an arc running almost in the Galactic plane from longitudes $150^{\circ}-300^{\circ}$. Although the charged particle induced background in the detectors produced a total count rate of 500-1000 counts $\mathrm{s}^{-1}$, the instrumental background rejection efficiency was such that the net count rate was $\sim 0.1 \mathrm{~s}^{-1}$ when the detectors were stowed, and $\sim 0.6$ $\mathrm{s}^{-1}$ when they were scanning the sky. Pre-flight modeling had shown that a net count rate of $\sim 0.5 \mathrm{~s}^{-1}$ was expected from this region of the sky so we have confidence that the DXS instrument was working as expected during its data collection times.

Figure 2 shows the data collected while DXS scanned the Galactic longitude range $213^{\circ}-252^{\circ}$, mostly in the constellation Puppis. The wavelength scale is still preliminary, as is the flux axis. The data do show lines, indicating that the source of the emission is thermal-we are indeed observing a hot interstellar medium. The solid line is the best-fit Raymond-Smith model folded through the detector response function. Both the temperature of the plasma and the amount of intervening absorbing material were allowed to float. Although the best-fit parameters are similar to those found by earlier experiments, $\mathrm{kT}=0.094\left(1.09 \times 10^{6} \mathrm{~K}\right)$ and $N_{\mathrm{H}}=6 \times 10^{19} \mathrm{~cm}^{-2}$, it is easy to see that the model does not fit the data. No single-temperature equilibrium plasma model (Raymond-Smith, Kaastra-Mewe, Landini-Fossi) in the temperature range $10^{5}-10^{6} \mathrm{~K}$ fits the data any better. We examined two-temperature models, and found that by combining a million-degree plasma and $\mathrm{a} \sim 2.5 \times 10^{6} \mathrm{~K}$ plasma we could do reasonably well in the DXS bandpass, but exceeded by factors of 3-4 the measured intensity in the $0.5-1 \mathrm{keV}$ range. Models with depleted elemental abundances were also tried, but without success. We conclude that either the models are seriously deficient, or that the emitting plasma is not in ionization equilibrium. We tried to fit the data with several non-equilibrium models, specifically blast wave models (Edgar \& Cox 1993) and cooling plasmas, both with solar elemental abundances and with depleted abundances. None of these fit any better than the single temperature equilibrium model. We may need to develop more appropriate models, incorporating new atomic physics data as they become available, and new astrophysical ideas.

We have compared the spectra from different regions of the sky with one another and our tentative conclusion is that we see small but probably significant differences in different directions. More analysis is needed to strengthen and quantify this conclusion.

The current analysis is focusing on completing the reduction and incorporation into the analysis models of all of the calibration data to better determine the wavelength scale, to better understand the detectors' efficiency at all wavelengths, and better remove any remaining instrumental artifacts in the data. Another facet of the current analysis is to place upper limits on the flux that could be present from individual ions as a function of temperature. The latter requires better models of the individual ionic spectra than are currently available from the standard models.

To summarize the state of spectroscopic observations of the hot interstellar medium:

- The low energy diffuse background shows evidence for lines, implying the emission is thermal.

- The detected spectra do not match model spectra of cosmic abundance equilibrium plasmas for $10^{5} \lessgtr \mathrm{T} \lesssim 10^{7} \mathrm{~K}$.

- We have not yet found a model with depleted abundances or a model of a nonequilibrium plasma that fits the data. We may need improved models.

- The data show weak evidence for different spectra in different directions, but further analysis is needed to confirm this conclusion.

- Additional data is needed, particularly with higher spectral resolution, higher angular resolution, and a wider range of directions on the sky. High latitude data would be very enlightening. 


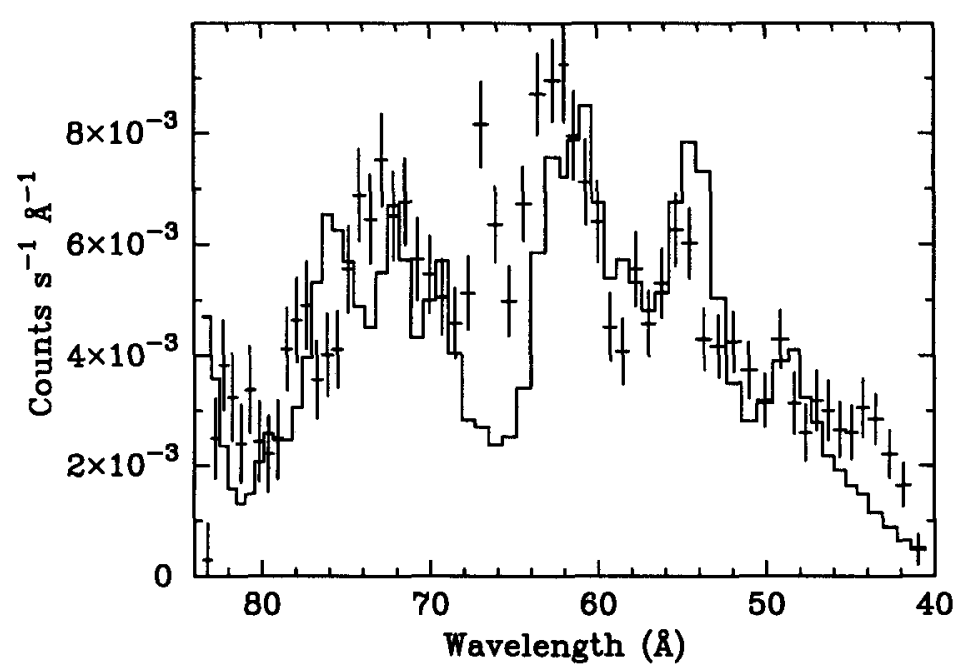

FIGURE 2. DXS data from the Galactic longitude range $213^{\circ}-252^{\circ}$. The solid line is the best-fit Raymond \& Smith model, temperture of $1.09 \times 10^{6} \mathrm{~K}, N_{\mathrm{H}}$ of $6 \times 10^{19} \mathrm{~cm}^{-2}$. The scales of both axes are preliminary.

The authors would like to acknowledge helpful discussions with Steve Snowden, Bill Kraushaar, and Dan McCammon in preparing this talk. This work was supported in part by NASA contract NAS 5-26078.

\section{REFERENCES}

Benjamin, R. A., Venn, K. A., Hrtgen, D. D., \& Sneden, C. 1995, The Distance to an $\mathrm{X}$-Ray Shadowing Molecular Cloud in Ursa Major. ApJ, submitted

Bloch, J. J., Jahoda, K., Juda, M., McCammon, D., Sanders, W. T., \& Snowden, S. L. 1986, Observations of the Soft X-Ray Background at $0.1 \mathrm{keV}, \mathrm{ApJ}, 308$, L59-L62

Bowyer, C. S., FIELD, G. B., \& MACK, J. E. 1968, Detection of an Anisotropic Soft X-ray Background Flux, Nature, 217, 32

Burrows, D. N., \& Mendenhall, J. A. 1991, Soft X-ray Shadowing by the Draco Cloud, Nature, 351, 629

Dickey, J. \& Lockman, F. J. 1990, H I in the Galaxy, Ann. Rev. Astr. Ap., 28, 215

Edgar, R. J. \& Cox, D. P. 1993, Hot Bubbles in a Magnetic Interstellar Medium: Another Look at the Soft X-ray Background, ApJ, 413, 190

EDwards, B. C. 1990, Observations of the Soft X-ray Background, PhD Thesis, University of Wisconsin, Madison

Garmire, G. P., Apparao, K. M. V., Burrows, D. N., Fink, R. L., \& Kraft, R. P. 1992, The Soft X-ray Diffuse Background Observed with the HEAO 1 Low-Energy Detectors, ApJ, 399, 694

Juda, M., Bloch, J. J., Edwards, B. C., McCammon, D., SANders, W. T., SNowden, S. L., \& ZhaNG, J. 1991, Limits on the Density of Neutral Gas within 100 Parsecs from 
Observations of the Soft X-Ray Background, ApJ, 367, 182

Marshall, F. J. \& Clark, G. W. 1984, SAS 9 Survey of the Soft X-ray Background, ApJ, 287,633

McCammon, D., Burrows, D. N., Sanders, W. T., \& Kraushaar, W. L. 1983, The Soft X-Ray Background, ApJ, 269, 107

Sanders, W. T., Edgar, R. J., Juda, M., Kraushaar, W. L., McCammon, D., Snowden, S. L., Zhang, J., \& SkInNer, M. A. 1992, The Diffuse X-ray Spectrometer Experiment, Proceeding of the SPIE: EUV, X-Ray, and Gamma-Ray Instrumentation for Astronomy III, 1743, 60

Snowden, S. L., Cox, D. P., McCammon, D., \& Sanders, W. T. 1990, A Model for the Distribution of Material Generating the Soft X-Ray Background, ApJ, 354, 211

Snowden, S. L., Mebold, U., Hirth, W., Herbstmeier, U., \& Schmitt, J. H. M. M. 1991, ROSAT Detection of an X-ray Shadow in the $\frac{1}{4} \mathrm{keV}$ Diffuse Background in the Draco Nebula, Science, 252, 1529

SNOwDEN, S. L. 1993, Implications of ROSAT Observations for the Local Hot Bubble, Adv. Space Res., 13(12), 103-111

Snowden, S. L., McCammon, D., \& Verter, F. 1993, The X-ray Shadow of the HighLatitude Molecular Cloud MBM 12, ApJ, 409, L21-L24

Snowden, S. L., Hasinger, G., Jahoda, K., Lockman, F. J., MCCammon, D., \& Sanders, W. T. 1994, Soft X-Ray and H I Surveys of the Low $N_{\mathrm{H}}$ Region in Ursa Major, ApJ, 430 , 601

Snowden, S. L., Freyberg, M. J., Plucinsky, P. P., Schmitt, J. H. M. M., TrüMPer, J., Voges, W., Edgar, R. J., McCammon, D., \& SAnders, W. T. 1995, First Maps of the Soft X-ray Diffuse Background from the ROSAT XRT/PSPC All-Sky Survey, ApJ, submitted 